\title{
A BASIS FOR RESIDUAL POLYNOMIALS IN $n$ VARIABLES*
}

\author{
BY \\ MARIE LITZINGER
}

\section{INTRODUCTION}

Kempner $\dagger$ has established the existence of a basis for residual polynomials in one variable with respect to a composite modulus. A residual polynomial modulo $m$ is by definition a polynomial $f(x)$ with integral coefficients which is divisible by $m$ for every integral value of $x$, and a residual congruence is written $f(x) \equiv 0(\bmod m)$. By a basis for a given modulus is meant a finite set of residual polynomials $p_{i}(x)$ which fulfills two requirements: (i) every residual polynomial modulo $m$ is expressible as a sum of products of $p_{i}(x)$ by polynomials in $x$ with integral coefficients; (ii) no member of the set $p_{i}(x)$ can be written identically equal to a sum of products of the remaining members of the set by polynomials in $x$ with integral coefficients.

For this work, the following notation is used. The symbol $\mu(d)$ denotes the least positive integer for which $d$ divides $\mu$ !. A special set of divisors of $m$ is chosen: separate all divisors of $m$ which exceed 1 into groups such that $\mu(d)$ has the same value for all the $d$ 's of a group but different values for the $d$ 's of different groups; select the largest $d$ of each group and denote this set by $d_{1}, \cdots, d_{s}$. Finally, $\Pi(\mu)=x(x-1) \cdots(x-\mu+1)$; when $x$ is replaced by $x_{i}$, the product will be designated by $\Pi_{j}(\mu) ; \Pi(1)$ is interpreted as 1 . Employing this notation, Dickson $\ddagger$ gave a brief proof of the theorem due to Kempner§:

Every residual polynomial $f(x)$ modulo $m$ is a sum of products of $m$ and $\left(m / d_{i}\right) \Pi\left(\mu\left(d_{i}\right)\right)$ for $i=1, \cdots, s$ by polynomials in $x$ with integral coefficients.

In a later paper, Kempner $\mathbb{T}$ considered the problem for $n$ variables. In attempting to apply Dickson's method to the proof of the existence of a basis for residual polynomials in more than one variable, I found that Kempner had omitted from the set $p_{i}\left(x_{1}, \cdots, x_{n}\right)$ certain residual polynomials in

* Presented to the Society, February 23, 1935; received by the editors July 8, 1934.

† These Transactions, vol. 22 (1921), pp. 240-266.

$\ddagger$ L. E. Dickson, Introduction to the Theory of Numbers, p. 26, Theorem 28.

§ These Transactions, vol. 22 (1921), p. 263.

IT These Transactions, vol. 27 (1925), pp. 287-298. 
several variables. This was brought to my attention by an example in two variables modulo 12 . For this modulus, the $d_{1}, \cdots, d_{\text {s }}$ are $d_{1}=12, d_{2}=6$, $d_{3}=2$; the corresponding $\mu$ 's are $\mu_{1}=4, \mu_{2}=3, \mu_{3}=2$. Write $q_{i}=m / d_{i}$. The part of the basis containing one variable is composed of

$$
\text { 12, } q_{i} \Pi_{1}\left(\mu_{i}\right), \quad q_{i} \Pi_{2}\left(\mu_{i}\right) \quad(i=1,2,3) .
$$

Kempner would include in the basis $p_{i}\left(x_{1}, x_{2}\right)$ modulo 12 only the seven terms (1). However, the residual polynomial,

$$
P=\left(m /\left(d_{3} \cdot d_{3}\right)\right) \Pi_{1}\left(\mu_{3}\right) \Pi_{2}\left(\mu_{3}\right)=3 x_{1}\left(x_{1}-1\right) x_{2}\left(x_{2}-1\right),
$$

must be added since, as is shown below, it is impossible to write the identity

$$
P=12 \cdot c+\sum_{i=1}^{3} q_{i} \Pi_{1}\left(\mu_{i}\right) f_{i}+\sum_{i=1}^{3} q_{i} \Pi_{2}\left(\mu_{i}\right) g_{i},
$$

where $c, f_{i}, g_{i}$ are polynomials in $x_{1}, x_{2}$ with integral coefficients. By use of $\left(x_{1}, x_{2}\right)=(0,0),(2,0),(0,2)$ we prove the constant terms of $c, f_{3}, g_{3}$ even. The pair $\left(x_{1}, x_{2}\right)=(2,2)$ shows the right side of (2) divisible by 24 and the left side equal to 12 .

\section{REPRESENTATION OF RESIDUAL POLYNOMIALS}

Dickson's method of establishing the existence of a basis for residual polynomials modulo $m$ in one variable may be applied to the case of two variables and then by induction to $n$ variables. Several preliminary steps are necessary. The first is the statement of two lemmas due to Dickson.

LEMMA* 1 . If $d$ is any divisor of $m, \mu(d)$ is the minimum degree of a residual polynomial $f(x)$ modulo $m$ whose leading coefficient is $m / d$.

LeMMa $\dagger$ 2. Any residual polynomial $f(x)$ modulo $m$ is term by term congruent modulo $m$ to the product of an integer prime to $m$ by a residual polynomial whose leading coefficient is a divisor of $m$.

The next step is to obtain a lemma similar to Lemma 2.

LEMMa 3. Any residual polynomial $f\left(x_{1}, \cdots, x_{n}\right)$ modulo $m$, written as a function of $x_{1}$ with coefficients containing $x_{2}, \cdots, x_{n}$, is term by term congruent modulo $m$ to the product of an integer prime to $m$ by a residual polynomial in which the greatest common divisor of the coefficients of the highest power of $x_{1}$ is a divisor of $m$.

Let

$$
f\left(x_{1}, \cdots, x_{n}\right)=c G\left(x_{2}, \cdots, x_{n}\right) x_{1}^{r}+\cdots,
$$

* L. E. Dickson, Introduction to the Theory of Numbers, p. 25, V.

† Ibid., p. 25, VI. 
where the coefficients of $G$ have the greatest common divisor 1 , and let $g$ be the greatest common divisor of $c=g C$ and $m=g M$. Since $C$ is prime to $M, C L \equiv 1(\bmod M)$ has a unique solution $L$. Then every integer satisfying $C z \equiv 1(\bmod M)$ is of the form $z=L+M y$, and $y$ can be chosen so that $z$ is prime to $m$. Consequently $z Z \equiv 1(\bmod m)$ has a solution $Z$ and $c z=g C z \equiv g$ $(\bmod m)$, also

$$
\begin{array}{rlrl}
z f & \equiv g G\left(x_{2}, \cdots, x_{n}\right) x_{1}{ }^{r}+\cdots & (\bmod m), \\
f \equiv Z\left[g G\left(x_{2}, \cdots, x_{n}\right) x_{1}{ }^{r}+\cdots\right] & (\bmod m) .
\end{array}
$$

Finally, two properties of $\mu$ and divisors of $m$ must be derived.

Lемма 4. If $d$ and $d^{\prime}$ are divisors of $m$ such that $d^{\prime}$ divides $d$, then $\mu\left(d^{\prime}\right) \leqq \mu(d)$.

Write $d=p_{1}^{a_{1}} \cdot p_{2}^{a_{2}} \cdots p_{k}{ }^{a_{k}}$ where $p_{1}, p_{2}, \cdots, p_{k}$ are distinct primes. Then $\mu(d)$ is the largest (or one of the largest in case several are equal)* of the numbers $\mu\left(p_{1}{ }^{a_{1}}\right), \mu\left(p_{2}{ }^{a_{2}}\right), \cdots, \mu\left(p_{k}{ }^{a_{k}}\right)$. Since $d^{\prime}$ divides $d, d^{\prime}=p_{1}{ }^{b_{1}}$ $\cdot p_{2}{ }^{b_{2}} \cdots p_{k}{ }^{b_{k}}$ where $0 \leqq b_{i} \leqq a_{i}$ for $i=1,2, \cdots, k$. So $\mu\left(p_{i}{ }^{b_{i}}\right) \leqq \mu\left(p_{i}{ }^{a_{i}}\right)$ for $i=1,2, \cdots, k$, and $\mu\left(d^{\prime}\right)$, the largest of the $\mu\left(p_{1}^{b_{1}}\right), \mu\left(p_{2}^{b_{2}}\right), \cdots, \mu\left(p_{k}{ }^{b_{k}}\right)$, is less than or equal to $\mu(d)$.

LEMMA 5 . If $d_{i}$ is one of the set $d_{1}, \cdots, d_{s}$ for $m$, then $d_{i}$ is divisible by every divisor of $m$ which divides $\mu\left(d_{i}\right)$ !.

The assumption that a divisor $d$ of $m$ divides $\mu\left(d_{i}\right)$ ! and does not divide $d_{i}$ leads to a contradiction as follows. Denote by $D$ the greatest common divisor of $d_{i}$ and $d$ so that $d_{i}=D D_{i}$ and $d=D D^{\prime}$. Since $\mu\left(d_{i}\right)$ ! is divisible by both $d_{i}$ and $d$ and since $D_{i}$ and $D^{\prime}$ are relatively prime, $\mu\left(d_{i}\right)$ ! is divisible by $N=D D_{i} D^{\prime}$ and $N$ divides $m$. As $N$ divides $\mu\left(d_{i}\right) !, \mu(N) \leqq \mu\left(d_{i}\right)$; as $N$ is divisible by $d_{i}$, by Lemma $4, \mu(N) \geqq \mu\left(d_{i}\right)$; consequently $\mu(N)=\mu\left(d_{i}\right)$. But $d_{i}$ is one of the set $d_{1}, \cdots, d_{s}$ and therefore is the maximum of all divisors $d_{i}$ of $m$ for which $\mu\left(d_{j}\right)=\mu\left(d_{i}\right)$. There is then a contradiction unless $D^{\prime}=1$, therefore $d$ divides $d_{i}$.

With the aid of these lemmas, it is possible to prove

THeorem 1. Every residual polynomial $f\left(x_{1}, x_{2}\right)$ modulo $m$ is a sum of products of $m$ and functions

$$
\left(m /\left(d_{i_{1}} \cdot d_{i_{2}}\right)\right) \Pi_{1}\left(\mu\left(d_{i_{1}}\right)\right) \Pi_{2}\left(\mu\left(d_{i_{2}}\right)\right)
$$

by polynomials in $x_{1}, x_{2}$ with integral coefficients, where $d_{i_{1}}, d_{i_{2}}$ are divisors of $m$, at least one belongs to the set $d_{1}, \cdots, d_{s}$, and the product $d_{i_{1}} \cdot d_{i_{2}}$ divides $m$.

By Lemma 3,

* Kempner, these Transactions, vol. 22 (1921), p. 243. 


$$
f\left(x_{1}, x_{2}\right)=m \phi\left(x_{1}, x_{2}\right)+Z F\left(x_{1}, x_{2}\right),
$$

where $Z$ and the coefficients of $\phi$ are integers, $Z$ is prime to $m$ and $F$ is a residual polynomial modulo $m$ of the form

$$
(m / d) G\left(x_{2}\right) x_{1}^{r}+\cdots,
$$

$d$ being a divisor of $m$. If $d=1$, the terms containing $m$ as a factor may be combined with $m \phi$ and the remaining portion considered the new $Z F$. So let $d>1$.

Case 1. Let $r \geqq \mu(d)$. Employ the relation*

$$
(m / d) \Pi_{1}(\mu(d))=\left(q m / d_{i_{1}}\right) \Pi_{1}\left(\mu\left(d_{i_{1}}\right)\right),
$$

where $d_{i_{1}}$ is one of the set $d_{1}, \cdots, d_{s}$ and $q$ is an integer. The product of (5) by $G\left(x_{2}\right) x_{1}^{r-\mu\left(d_{i_{1}}\right)}$ gives a function whose term in $x_{1}{ }^{r}$ is identical with that of $F$. The difference is a residual polynomial of degree less than $r$ in $x_{1}$.

Case 2. Let $r<\mu(d)$. Consider $F$ which is of the form (4). For a chosen value $x_{2}^{\prime}$ of $x_{2}$, by Lemma $2, F$ as a residual polynomial in $x_{1}$ is term by term congruent modulo $m$ to the product of an integer prime to $m$ by a residual polynomial whose leading coefficient is a divisor of $m$, that is,

$$
F\left(x_{1}, x_{2}^{\prime}\right)=(m / d) G\left(x_{2}^{\prime}\right) x_{1}^{r}+\cdots \equiv z\left(\left(m / d^{\prime}\right) x_{1}^{r}+\cdots\right) \quad(\bmod m),
$$

where $z$ is prime to $m$ and $d^{\prime}$ divides $m$. Then $(m / d) G\left(x_{2}{ }^{\prime}\right)=z \cdot m / d^{\prime}+k m$ where $k$ is integral. As $m / d^{\prime}$ divides $m,(m / d) G\left(x_{2}^{\prime}\right)$ is divisible by $m / d^{\prime}$. Now $\left(m / d^{\prime}\right) x_{1}{ }^{r}+\cdots$ is a residual polynomial whose leading coefficient is a divisor of $m$, consequently, by Lemma $1, r \geqq \mu\left(d^{\prime}\right)$. Let $d_{i}$ represent the divisor of the set $d_{1}, \cdots, d_{s}$ to which corresponds the largest $\mu$ which does not exceed $r$. Then $\mu\left(d_{i}\right) \geqq \mu\left(d^{\prime}\right)$, therefore $d^{\prime}$ divides $\mu\left(d_{i}\right)$ !. By Lemma 5 , $d^{\prime}$ divides $d_{i}$. Consequently $m / d_{i}$ divides $m / d^{\prime}$ and must then divide $(m / d) G\left(x_{2}{ }^{\prime}\right)$.

There is an important consequence of the divisibility of $(m / d) G\left(x_{2}^{\prime}\right)$ by $m / d_{i}$. Note first that $m / d_{i}$ does not divide $m / d$, for if $d$ divides $\dot{d}_{i}$, by Lemma $4, \mu(d) \leqq \mu\left(d_{i}\right)$; but by the definition of $d_{i}, \mu\left(d_{i}\right) \leqq r$; the conclusion $\mu(d) \leqq r$ contradicts the hypothesis of this second case, namely $r<\mu(d)$. Since $m / d_{i}$ does not divide $m / d$, denote their greatest common divisor by $M$. Then

$$
m / d_{i}=M g, m / d=M v, v \cdot m / d_{i}=g \cdot m / d,
$$

where $g>1$ and prime to $v$. From the divisibility of $(m / d) G\left(x_{2}^{\prime}\right)$ by $m / d_{i}$, it follows that the quotient of $(m / d) G\left(x_{2}^{\prime}\right)$ by $m / d_{i}$, which equals $(v / g) G\left(x_{2}{ }^{\prime}\right)$, is integral. As $g$ is prime to $v, g$ divides $G\left(x_{2}^{\prime}\right)$.

Consider $G\left(x_{2}\right)$ for other values of $x_{2}$. Although the coefficient correspond-

${ }^{*}$ L. E. Dickson, Introduction to the Theory of Numbers, p. 27, equation (34). 
ing to $m / d^{\prime}$ varies with the choice of $x_{2}, d_{i}$ by definition is determined by $r$ and $m$ and is independent of the value of $x_{2}$. Consequently $g$ and $v$, determined by $m / d_{i}$ and $m / d$, do not vary with $x_{2}$. So for every choice of $x_{2}$, the $m / d^{\prime}$ determined by it is such that it divides $(m / d) G\left(x_{2}\right)$ and is divisible by $m / d_{i}$; therefore $(m / d) G\left(x_{2}\right)$ is divisible by $m / d_{i}$ and $G\left(x_{2}\right)$ is divisible by $g$.

Since $G\left(x_{2}\right)$ is divisible by $g$ for every value of $x_{2}, G\left(x_{2}\right) \equiv 0(\bmod g)$. Therefore $G\left(x_{2}\right)$ is expressible* as a sum of products of $g$ and $\left(g / d_{i_{2}}\right) \Pi_{2}\left(\mu\left(d_{i_{2}}\right)\right)$ by polynomials in $x_{2}$ with integral coefficients, where the $d_{i_{2}}$ represent the set of divisors of $g$ selected as the set $d_{1}, \cdots, d_{s}$ was chosen from all divisors of $m$. As $g$ divides $m$, for each $d_{i_{2}}, \mu\left(d_{i_{2}}\right)=\mu\left(d_{h}\right)$ where $d_{h}$ is one of the set $d_{1}, \cdots, d_{s}$ for $m$ and, by Lemma $5, d_{i_{2}}$ equals or divides $d_{h}$.

In the work which follows, write $d_{i_{1}}$ for $d_{i}$ to indicate its association with $x_{1}$. The term of $F$ containing the highest power of $x_{1}$ may be expressed as follows:

$$
(m / d) G\left(x_{2}\right) x_{1}^{r}=g(m / d)(1 / g) G\left(x_{2}\right) x_{1}^{r}=v\left(m / d_{i_{1}}\right)(t / m) G\left(x_{2}\right) x_{1}^{r},
$$

where $t$ is defined by the equation $t g=m$, and $g$ and $v$ are defined by (6). Note that $d_{i_{1}}$ divides $t$ from the following considerations: $t / d_{i_{1}}=(m / g) g /(d v)$ $=m /(d v)$ which is integral since $v$ divides $m / d$. As $d_{i_{1}}$ divides $t$ and each $d_{i_{2}}$ is a factor of $g$, for every $d_{i_{2}}$, the product $d_{i_{1}} d_{i_{2}}$ divides $m$. From its definition, $d_{i_{1}}$ is one of the set $d_{1}, \cdots, d_{s}$ for $m$. The product of $v\left(m / d_{i_{1}}\right) \Pi_{1}\left(\mu\left(d_{i_{1}}\right)\right)$ by $(t / m) G\left(x_{2}\right) x_{1}{ }^{r-\mu\left(d_{\left.i_{1}\right)}\right)}$ gives a function whose term in $x_{1}{ }^{r}$ is identical with that of $F$. The difference is a residual polynomial of degree less than $r$ in $x_{1}$.

This process, continued for the resulting polynomials considered as functions of $x_{1}$ or $x_{2}$, lowers the degree in $x_{1}$ or $x_{2}$ at each step and leads to a difference zero. Finally $f\left(x_{1}, x_{2}\right)$ is expressed in the manner described in Theorem 1 .

A similar theorem for $n$ variables is readily proved by induction.

THEOREM 2. Every residual polynomial $f\left(x_{1}, \cdots, x_{n}\right)$ modulo $m$ is a sum of products of $m$ and functions

$$
\left(m /\left(d_{i_{1}} \cdots d_{i_{n}}\right)\right) \Pi_{1}\left(\mu\left(d_{i_{1}}\right)\right) \cdots \Pi_{n}\left(\mu\left(d_{i_{n}}\right)\right)
$$

by polynomials in $x_{1}, \cdots, x_{n}$ with integral coefficients where the $d_{i_{j}}$ are divisors of $m$, at least one of the $d_{i_{1}}, \cdots, d_{i_{n}}$ belongs to the set $d_{1}, \cdots, d_{s}$, and the product $d_{i_{1}} \cdots d_{i_{n}}$ divides $m$.

The theorem has been established for the case $n=2$. Assume that it holds for $n-1$ variables and show that it must then be true for $n$. By Lemma 3,

$$
f\left(x_{1}, \cdots, x_{n}\right)=m \phi\left(x_{1}, \cdots, x_{n}\right)+Z F\left(x_{1}, \cdots, x_{n}\right),
$$

where $Z$ and the coefficients of $\phi$ are integers, $Z$ is prime to $m$, and $F$ is a resid-

* L. E. Dickson, Introduction to the Theory of Numbers, p. 26, Theorem 28. 
ual polynomial modulo $m$ of the form

$$
(m / d) G\left(x_{2}, \cdots, x_{n}\right) x_{1}^{r}+\cdots,
$$

$d$ being a divisor of $m$. If $d=1$, the terms containing $m$ as a factor may be combined with $m \phi$ and the remaining portion considered the new $Z F$. So let $d>1$.

Case 1. Let $r \geqq \mu(d)$. Employ relation (5). The product of (5) by $G\left(x_{2}, \cdots, x_{n}\right) x_{1}^{r-\mu\left(d_{i_{1}}\right)}$ gives a function whose term in $x_{1}{ }^{r}$ is identical with that of $F$. The difference is a residual polynomial modulo $m$ of degree less than $r$ in $x_{1}$.

Case 2. Let $r<\mu(d)$. Consider $F$ which is of the form (8). For a chosen set of values $x_{2}^{\prime}, \cdots, x_{n}^{\prime}$, by Lemma $2, F$ as a residual polynomial in $x_{1}$ modulo $m$ is term by term congruent modulo $m$ to the product of an integer prime to $m$ by a residual polynomial whose leading coefficient is a divisor of $m$, that is,

$$
\begin{aligned}
F\left(x_{1}, x_{2}^{\prime}, \cdots, x_{n}^{\prime}\right) & =(m / d) G\left(x_{2}^{\prime}, \cdots, x_{n}^{\prime}\right) x_{1}^{r}+\cdots \\
& \equiv z\left(\left(m / d^{\prime}\right) x_{1}^{r}+\cdots\right) \quad(\bmod m),
\end{aligned}
$$

where $z$ is prime to $m$ and $d^{\prime}$ divides $m$. For the chosen set $x_{2}^{\prime}, \cdots, x_{n}^{\prime}$, $(m / d) G\left(x_{2}^{\prime}, \cdots, x_{n}^{\prime}\right)=z \cdot m / d^{\prime}+k m$ where $k$ is integral. As $m / d^{\prime}$ divides $m$, $m / d^{\prime}$ divides $(m / d) G\left(x_{2}^{\prime}, \cdots, x_{n}^{\prime}\right)$. Now $\left(m / d^{\prime}\right) x_{1}{ }^{r}+\cdots$ is a residual polynomial whose leading coefficient is a divisor of $m$, consequently, by Lemma $1, r \geqq \mu\left(d^{\prime}\right)$.

Repeat the argument given in Theorem 1 for Case 2, defining $d_{i}$ as the divisor of the set $d_{1}, \cdots, d_{s}$ for $m$ to which corresponds the largest $\mu$ not exceeding $r$, and replacing the phrase "value of $x_{2}^{\prime \prime}$ " by "set of values $x_{2}^{\prime}, \cdots, x_{n}^{\prime}$," and $G\left(x_{2}^{\prime}\right)$ by $G\left(x_{2}^{\prime}, \cdots, x_{n}^{\prime}\right)$. Exactly as in the first two paragraphs of Case 2, Theorem $1, m / d_{i}$ divides $m / d^{\prime}$ and therefore divides $(m / d) G\left(x_{2}^{\prime}, \cdots, x_{n}^{\prime}\right)$; but $m / d_{i}$ does not divide $m / d$. Let $M$ denote the greatest common divisor of $m / d_{i}$ and $m / d$, and obtain (6). Then the quotient of $(m / d) G\left(x_{2}^{\prime}, \cdots, x_{n}^{\prime}\right)$ by $m / d_{i}$, which equals $(v / g) G\left(x_{2}^{\prime}, \cdots, x_{n}^{\prime}\right)$, is integral. Since $g$ is prime to $v, g$ divides $G\left(x_{2}^{\prime}, \cdots, x_{n}^{\prime}\right)$.

Consider $G\left(x_{2}, \cdots, x_{n}\right)$ for other values of $x_{2}, \cdots, x_{n}$. Although the coefficient corresponding to $m / d^{\prime}$ varies with the choice of $x_{2}, \cdots, x_{n}, d_{i}$ is determined by $r$ and $m$ and is independent of the values of $x_{2}, \cdots, x_{n}$. Consequently: $g$ and $v$, determined by $m / d_{i}$ and $m / d$, do not vary with $x_{2}, \cdots, x_{n}$. So for every choice of $x_{2}, \cdots, x_{n}$, the $m / d^{\prime}$ determined by it is such that it divides $(m / d) G\left(x_{2}, \cdots, x_{n}\right)$ and is divisible by $m / d_{i}$; therefore $(m / d) G\left(x_{2}, \cdots, x_{n}\right)$ is divisible by $m / d_{i}$ and $G\left(x_{2}, \cdots, x_{n}\right)$ is divisible by $g$.

Since $G\left(x_{2}, \cdots, x_{n}\right)$ is divisible by $g$ for every set of values $x_{2}, \cdots, x_{n}$, $G\left(x_{2}, \cdots, x_{n}\right) \equiv 0(\bmod g)$. According to the hypothesis, $G$ as a residual 
polynomial in $n-1$ variables is expressible as a sum of products of $g$ and

$$
\left(g /\left(d_{i_{2}} \cdots d_{i_{n}}\right)\right) \Pi_{2}\left(\mu\left(d_{i_{2}}\right)\right) \cdots \Pi_{n}\left(\mu\left(d_{i_{n}}\right)\right)
$$

by polynomials in $x_{2}, \cdots, x_{n}$ with integral coefficients, where the $d_{i,}$ (for $j=2, \cdots, n)$ represent divisors of $g$ and $d_{i_{2}} \cdots d_{i_{n}}$ divides $g$. Since $g$ divides $m$, for each $d_{i_{j}}, \mu\left(d_{i_{j}}\right)=\mu\left(d_{h}\right)$ where $d_{h}$ is one of the set $d_{1}, \cdots, d_{t}$ for $m$, and, by Lemma $5, d_{i j}$ equals or divides $d_{h}$.

For the following work, write $d_{i_{1}}$ in place of $d_{i}$ to indicate its association with $x_{1}$. The term of $F$ which contains the highest power of $x_{1}$ may be expressed as follows:

$$
(m / d) G\left(x_{2}, \cdots, x_{n}\right) x_{1}{ }^{r}=v\left(m / d_{i_{1}}\right)(t / m) G\left(x_{2}, \cdots, x_{n}\right) x_{1}^{r},
$$

where $t$ is defined by the equation $t g=m$, and $g$ and $v$ are defined by (6). As in the fifth paragraph of Case 2, Theorem 1, $d_{i_{1}}$ divides $t$. Since each product $d_{i_{2}} \cdots d_{i_{n}}$ divides $g$, then $d_{i_{1}} d_{i_{2}} \cdots d_{i_{n}}$ divides $m$. The product of $v\left(m / d_{i_{1}}\right)$ $\Pi_{1}\left(\mu\left(d_{i_{1}}\right)\right)$ by $(t / m) G\left(x_{2}, \cdots, x_{n}\right) x_{1}{ }^{r-\mu\left(d_{i_{1}}\right)}$ gives a function whose term in $x_{1}{ }^{r}$ is identical with that of $F$. The difference is a residual polynomial of degree less than $r$ in $x_{1}$.

This process, continued for the resulting polynomials considered as functions of $x_{j}$ for $j=1,2, \cdots, n$, lowers the degree in $x_{j}$ at each step and leads to a difference zero. Finally $f\left(x_{1}, \cdots, x_{n}\right)$ is expressed in the manner described in Theorem 2.

Theorems 1 and 2 contain one interesting difference from the theorem for one variable. Of the divisors of $m$ appearing in a term (3) or (7), only one is necessarily chosen from $d_{1}, \cdots, d_{s}$.

\section{Selection of a BASIS}

It is now essential to establish a basis for residual polynomials in $n$ variables modulo $m$. By Theorem 2, the set composed of $m$ and all terms (7) fulfills the first requirement for a basis. It remains to select from $m$ and (7) a reduced set $p_{i}\left(x_{1}, \cdots, x_{n}\right)$ such that no member of $p_{i}$ can be written identically equal to a sum of products of the remaining $p_{i}$ by polynomials in $x_{1}, \cdots, x_{n}$ with integral coefficients, and such that each of the terms of $m$ and (7) not included among the $p_{i}$ can be written identically equal to a sum of products of $p_{i}$ by polynomials in $x_{1}, \cdots, x_{n}$ with integral coefficients. The terms $p_{i}$ form a basis and will be called independent. All other terms $m$ and (7) will be called dependent.

A term $k \cdot \Pi_{1}\left(\mu\left(d_{i_{1}}\right)\right) \cdots \Pi_{n}\left(\mu\left(d_{i_{n}}\right)\right)$ of $(7)$ whose coefficient $k$ is a multiple of that of another term (7) containing $\Pi_{1}\left(\mu\left(d_{i_{1}}\right)\right) \cdots \Pi_{n}\left(\mu\left(d_{i_{n}}\right)\right)$ is obviously dependent. Discard such terms and represent the remaining terms (7) by 


$$
P\left(d_{i_{1}}, \cdots, d_{i_{n}}\right) \text {. }
$$

Denote by $S$ the set composed of $m$ and all terms (9). Throughout the discussion, an element of the set $S$ will be termed simple or compound according as it contains one variable or more than one variable. The phrase "member related to (9)" will be used to designate any term of the set $S$, simple or compound, which contains not more than $\mu\left(d_{i_{j}}\right)$ factors in $x_{j}$ for each $j=1, \cdots, n$.

The following theorem establishes a basis.

Theorem 3. For the general modulus $m$, a basis for residual polynomials in $n$ variables is composed of $m$, all simple terms and all compound terms (9) such that $\mu\left(d_{i_{1}}\right), \cdots, \mu\left(d_{i_{n}}\right)$ are all multiples of the same prime factor of $m$.

Example. For the modulus $3^{3} \cdot 5$, the set $S$ in two variables contains terms which are not members of the basis. The $d_{1}, \cdots, d_{\text {s }}$ for this modulus are $d_{1}=3^{3} \cdot 5, d_{2}=3^{2} \cdot 5, d_{3}=3 \cdot 5, d_{4}=3$; the corresponding $\mu^{\prime}$ s are $\mu_{1}=9, \mu_{2}=6$, $\mu_{3}=5, \mu_{4}=3$. The basis is composed of $3^{3} \cdot 5$, all simple terms, and the compound terms $P\left(d_{4}, d_{4}\right), P\left(d_{4}, d_{2}\right), P\left(d_{2}, d_{4}\right)$. The dependent compound terms of $S$ are expressible in terms of the basis as follows:

$$
\begin{aligned}
& P\left(d_{4}, d_{3}\right)=2\left(x_{2}-3\right)\left(x_{2}-4\right) P\left(d_{4}, d_{4}\right)-3 x_{1}\left(x_{1}-1\right)\left(x_{1}-2\right) q_{3} \Pi_{2}\left(\mu_{3}\right), \\
& P\left(d_{3}, d_{4}\right)=2\left(x_{1}-3\right)\left(x_{1}-4\right) P\left(d_{4}, d_{4}\right)-3 x_{2}\left(x_{2}-1\right)\left(x_{2}-2\right) q_{3} \Pi_{1}\left(\mu_{3}\right) .
\end{aligned}
$$

The part of Theorem 3 concerned with simple terms is readily established. Kempner* proved that $m$ and $\left(m / d_{i}\right) \Pi\left(\mu\left(d_{i}\right)\right)$ for $i=1, \ldots, s$ form a basis for residual polynomials modulo $m$ in one variable. It follows that $m$ as well as each simple term of $S$ is independent of all other members of the set $S$. For instance, to show the independence of a simple term in $x_{j}$, set the remaining $n-1$ variables equal to zero.

The proof of the portion of Theorem 3 which deals with compound terms will be divided into two parts. First it will be shown that each of the compound terms listed in the theorem is independent of all other members of the set $S$. Then it will be shown by means of an auxiliary theorem that these are the only independent compound terms.

It is not difficult to prove the independence of the compound terms described in Theorem 3. Suppose it were possible to write the identity

$$
P\left(d_{i_{1}}, \cdots, d_{i n}\right)=m \cdot c+\sum_{i} P_{i} \cdot f_{i},
$$

where $c$ and $f_{i}$ are polynomials in $x_{1}, \cdots, x_{n}$ with integral coefficients and the $P_{i}$ represent all members of the set $S$, simple and compound, except

$$
P\left(d_{i_{1}}, \cdots, d_{i_{n}}\right) \text {. }
$$

* These Transactions, vol. 22 (1921), pp. 263-264. 
By hypothesis each $\mu$ on the left side of (10) is a multiple of a prime $p$ which divides $m$. That each term on the right contains one more factor $p$ than appears on the left for $x_{1}=\mu\left(d_{i_{1}}\right), \cdots, x_{n}=\mu\left(d_{i_{n}}\right)$ may be shown as follows. Substitute successively for each $x_{j}$ the values $0, \mu\left(d_{i_{k}}\right)$ for all $k=1, \cdots, n$ such that $\mu\left(d_{i_{k}}\right) \leqq \mu\left(d_{i_{j}}\right)$. Under this substitution, terms $P_{i}$ not related to (11) disappear, and the constant term of $c$ and each remaining $f_{i}$ associated with a member of the basis containing only $\mu$ 's which are multiples of $p$ is proved congruent to zero modulo $p$. Related members not included among the latter present no difficulty since for the values listed above each will contain $p$ to a power at least one greater than that exhibited in the modulus, $p^{s}$. For instance, consider the simple term $\left(m / d_{i}\right) \Pi\left(\left(\mu\left(d_{i}\right)\right)\right.$ which lies between terms whose variable parts are $\Pi(r p)$ and $\Pi((r+1) p)$ and contain respectively $r p$ and $(r+1) p$ factors. This implies $r p<\mu\left(d_{i}\right)<(r+1) p$. For $x=r p,\left(m / d_{i}\right)$ $\cdot \Pi\left(\mu\left(d_{i}\right)\right)$ is zero; for $x=\mu\left(d_{i}\right)$ it is divisible by exactly $p^{s}$. The sequence $\Pi\left(\mu\left(d_{i}\right)\right)=x(x-1) \cdots\left(x-\mu\left(d_{i}\right)+1\right)$ contains at least one higher power of $p$ for $x=(r+1) p$ than for $x=\mu\left(d_{i}\right)$ since one additional factor $p$ is thus introduced at the beginning of the sequence when $(r+1) p$ is substituted for $x$, and none is lost at the end as the sequence contains more than $r p$ factors. The same reasoning holds for members of the set $S$ formed by compounding $\left(m / d_{i}\right) \Pi\left(\mu\left(d_{i}\right)\right)$ with other terms; however it will be shown in the Auxiliary Theorem that such members are dependent. The independence of (11) follows immediately; substitute for each $x_{j}$ the value $\mu\left(d_{i_{j}}\right)$. The left side of (10) is divisible by exactly $p^{*}$, the right side by $p^{*+1}$.

It is possible to select from the set $S$ certain dependent terms. If the coefficient of a compound member is the greatest common divisor of the coefficients of related terms, it is expressible as a linear homogeneous function of them with integral coefficients. Since the related terms by definition contain no more factors in any one variable than appear in the given term, the latter may be expressed by means of the related members in the manner described in Theorem 3.

That these are the only dependent terms follows from the

Auxiliary Theorem. For a compound term (11) of the set $S$ for $n$ variables modulo $m$, if

$$
m /\left(d_{i_{1}} \cdots d_{i_{n}}\right)
$$

is not the greatest common divisor of the coefficients of all related members of the set $S$, each $\mu\left(d_{i_{j}}\right)$ for $j=1, \cdots, n$ is a multiple of the same prime factor of the modulus.

Adopt the following notation to designate terms related to (11): let $d_{i_{j}}{ }^{\prime}$ represent any of the divisors of $m$ such that $\mu\left(d_{i_{j}}^{\prime}\right)<\mu\left(d_{i_{j}}\right)$ for $j=1, \cdots, n$. 
Then the coefficient of any term related to (11) is of the form

$$
m /\left(d_{k_{1}} \cdots d_{k_{n}}\right) \text {, }
$$

where at least one $d_{k_{j}}=d_{i_{j}}{ }^{\prime}$ if all of them are greater than 1 ; for one possible type of coefficients (13), each $d_{k_{i}}=d_{i_{i}}{ }^{\prime}$.

From the manner in which the set $S$ is constructed, (12) divides the coefficients of all related terms. Since by hypothesis (12) is not the greatest common divisor of all coefficients (13), denote their greatest common divisor by the product of $k$ by (12), where $k$ is a divisor of $m$ greater than 1 which may or may not be prime to (12). Then for $j=1, \cdots, n$, each $m / d_{i_{j}^{\prime}}$ contains a higher power of $k$ than appears in $m / d_{i_{j}}$. Otherwise one of the combinations (13) would equal (12) and (12) would be the greatest common divisor of all coefficients of related terms. In other words, the coefficient of every simple term of the set $S$ which exceeds $m / d_{i_{j}}$ contains a higher power of $k$ than does $m / d_{i_{j}}$. Since for each $j$, the largest $\Pi_{j}\left(\mu\left(d_{i_{j}}^{\prime}\right)\right)$ has a coefficient which divides all the other $m / d_{i_{j}}^{\prime}$, its coefficient contains the product of $k$ by (12). So for each $j, \Pi_{j}\left(\mu\left(d_{i_{j}}\right)\right)$ differs from the largest $\Pi_{j}\left(\mu\left(d_{i_{j}}{ }^{\prime}\right)\right)$ in that it is divisible by a higher power of $k$ than $\Pi_{j}\left(\mu\left(d_{i_{j}}^{\prime}\right)\right)$ for all values of $x_{j}$. Therefore if $k$ is a prime $p$, for each $j, \mu\left(d_{i_{j}}\right)$ is a multiple of $p$; if $k$ is composite, for each $j$, $\mu\left(d_{i_{j}}\right)$ is a multiple of the same prime factor of $k$.

There are two corollaries to Theorem 3.

COROLlaRY 1. For a modulus composed of the product of distinct primes, a basis is composed of $m$ and all simple terms.

Write the modulus $m$ as $p_{1} p_{2} \cdots p_{c}$ where the $p^{\prime}$ s are distinct primes arranged in descending order. The set $d_{1}, \cdots, d_{s}$ for $m$ is composed of $p_{1} p_{2} p_{3} \cdots p_{c}, p_{2} p_{3} \cdots p_{c}, \cdots, p_{c-1} p_{c}, p_{c}$; the corresponding $\mu$ 's are $p_{1}$, $p_{2}, \cdots, p_{c-1}, p_{c}$. No product of two or more of the $d$ 's listed above will divide $m$ since each contains $p_{c}$. Even when all possible divisors of $m$ are considered, as each one associated with $\mu=p_{j}$ contains $p_{j}$ as a factor, the product of two or more such divisors, if it divides $m$, divides one of the $d$ 's given above. Consequently a residual polynomial whose coefficient is $m$ divided by this product is expressible as a single variable member of the set $S$ multiplied by a polynomial with integral coefficients.

CoRollary 2. For a modulus equal to the power of a prime, a basis is composed of all terms $m$ and (9).

The divisors of $m=p^{k}$ are powers of $p$, and the $\mu$ 's are all multiples of $p$.

Mount Holyoke College,

South Hadley, Mass. 